\title{
Rates of shear in coaxial cylinder viscometers
}

By R. K. Code and J. D. Raal (Kingston)

Rheol. Acta 12, 494-503 (1973)

p. 495, Eq. [6],

read correctly:

$r^{2} d \tau+2 r \tau d r=0$

p. 501, Eq. [31], read correctly:

$\left.\Omega\right|_{s^{2} p_{\tau_{1}}}=\left.\Omega\right|_{\tau_{1}} \cdot e^{\frac{m_{1}}{\alpha}[s p \alpha-1]}$.

Eq. [32],

read correctly:

$f\left(\tau_{1}\right)=2 \Omega_{1} \cdot m_{1} \sum_{p=0}^{\infty} e^{\frac{m_{1}}{\alpha}\left[s^{2 p \alpha}-1\right]} \cdot s^{2 p \alpha}$

p. 502, Eq. [33],

read correctly:

$C_{R \alpha}=m_{1}\left(1-s^{2}\right) \sum_{p=0}^{\infty} e^{\frac{m_{1}}{\alpha}\left[s^{2 p x}-1\right]} \cdot s^{2 p \alpha}$.
Eq. [42],

read correctly:

$$
\begin{aligned}
\sum_{p=0}^{\infty} s^{2 p|\alpha|} & -\sum_{p=0}^{N} s^{2 p|x|} \\
& =\frac{1}{1-s^{2 \alpha}}-\sum_{p=0}^{N} s^{2 p|\alpha|}<10^{-5} .
\end{aligned}
$$

Eq. [43],

read correctly:

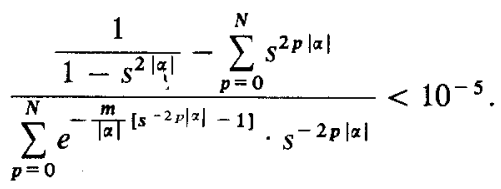

p. 503, Summary, right column, second eq., read correctly:

$C_{R}=m\left(1-s^{2}\right) \sum_{p=0}^{\infty} e^{\frac{m}{\alpha}\left[s^{2 p x}-1\right]} \cdot s^{2 p \alpha}$.

\section{Comparison of the time-temperature superposition of the relaxation modulus and time-to-break of preswollen gels}

\author{
By J. Janáček, M. Raab and M. Stol (Prague)
}

Rheol. Acta 13, 624-634 (1974)

p. 625, table 1 ,

read correctly:

Table 1. Swelling degrees $v_{2}$ and porosities $\mathrm{v}_{x}$ of PHEMA gels

$\begin{array}{lll}v_{0} \quad \text { Preswollen in water } & \begin{array}{l}\text { Preswollen } \\ \text { in ethylene } \\ \text { glycol }\end{array} \\ & \text { Equilibrium swollen in water } & \begin{array}{l}\text { Swollen } \\ \text { in ethylene } \\ \text { glycol }\end{array}\end{array}$

Concentration of the crosslinking agent

$c \times 10^{4} \mathrm{~mol} \mathrm{~cm}^{-3}$

$\begin{array}{llll}0.21 & 0.65 & 0.21 & 0.65\end{array}$

\begin{tabular}{lllllll} 
& $v_{2}$ & $v_{x}$ & $v_{2}$ & $v_{x}$ & $v_{2}$ & $v_{2}$ \\
\hline 0.8 & 0.529 & 1.0 & 0.541 & 1.0 & - & - \\
0.6 & - & - & - & - & 0.6 & 0.6 \\
0.5 & 0.518 & 0.98 & 0.523 & 0.97 & 0.5 & 0.5 \\
0.4 & 0.487 & 0.92 & 0.487 & 0.90 & 0.4 & 0.4 \\
0.3 & 0.356 & 0.67 & 0.384 & 0.71 & 0.3 & 0.3 \\
0.2 & 0.198 & 0.37 & 0.274 & 0.51 & - & -
\end{tabular}

